\title{
High Aspect Ratio Micromirrors With Large Static Rotation and Piston Actuation
}

\author{
Veljko Milanović, Member, IEEE, Sunghoon Kwon, and Luke P. Lee
}

\begin{abstract}
Monolithic high aspect ratio silicon micromirror devices were demonstrated with fully isolated vertical comb drives micromachined from the back side and front side of a 50- $\mu \mathrm{m}$ silicon-on-insulator device layer. The devices are suspended by torsional support beams and exhibit bidirectional single-axis rotation, as well as independent up- and down-pistoning actuation. The comb drives are fabricated with initial comb-finger preengagement of up to $10 \mu \mathrm{m}$ for constant force actuation and have accurately self-aligned comb-fingers. Device 1 with a $30-\mu \mathrm{m}$-thick support beam measured static optical beam deflection from $-20^{\circ}$ to $19^{\circ}$ and bidirectional pistoning motion from -7.5 to $8.25 \mu \mathrm{m}$. Device 2 utilizes a highly compliant 10 - $\mu$ m-thick support beam and measured static optical beam deflection from $-14^{\circ}$ to $16^{\circ}$ and downward pistoning motion to $-\mathbf{1 2 . 5} \mu \mathrm{m}$, all at $<70 \mathrm{~V}$.
\end{abstract}

Index Terms-Comb drive, micromirror, high aspect ratio, microelectromechanical systems (MEMS), microfabrication, micromachining, optical microelectromechanical systems (MEMS).

\section{INTRODUCTION}

$\mathbf{S}$ ILICON-ON-INSULATOR (SOI)-based microelectromechanical systems (MEMS) have become increasingly interesting recently as a platform for a variety of optical applications [1]-[10]. Traditionally, however, SOI-MEMS actuators have provided only in-wafer-plane motion. Thus, for many optical applications such as scanning micromirrors, a variety of methodologies are investigated to provide the needed additional, out-of-wafer-plane degrees of freedom (DoF). Particularly of interest is providing $1 \mathrm{DoF}$ (or single-axis) and $2 \mathrm{DoF}$ (two-axis) rotation of micromirrors. There is also demand for micromirrors with independently controlled rotation and pistoning motion [11]. We previously demonstrated laterally actuated micromirror structures with large static optical rotation of $\pm 20^{\circ}$ by fabricating vertically displaced beams (Fig. 1), [5], [6]. However, to address the need for the combination of pistoning with rotation, our previous [5] fabrication methodology required improvements.

Of interest was to add vertical comb drives. Vertically staggered SOI comb drives perform well for single-sided rotation applications [2], [3], [8] and demonstrated advantages of SOI-MEMS such as flatness, speed, and robustness, with respect to surface-micromachined examples of vertical comb drives [11], [12]. However, in these previous SOI comb drive processes, no isolation is available between comb drive fingers in either upper or lower comb drives, thus, one-sided rotation was demonstrated. Namely, in these previous methodologies, lower comb-fingers are etched into the handle wafer of SOI and, therefore, at the same potential and mechanically fixed, while all upper comb-fingers

Manuscript received September 26, 2003; revised March 20, 2004.

V. Milanović is with the Adriatic Research Institute, Berkeley, CA 947091070 USA (e-mail: veljko@adriaticresearch.org).

S. Kwon and L. P. Lee are with the Department of Bioengineering, Berkeley Sensor and Actuator Center, University of California, Berkeley, CA 94720 USA.

Digital Object Identifier 10.1109/LPT.2004.828351

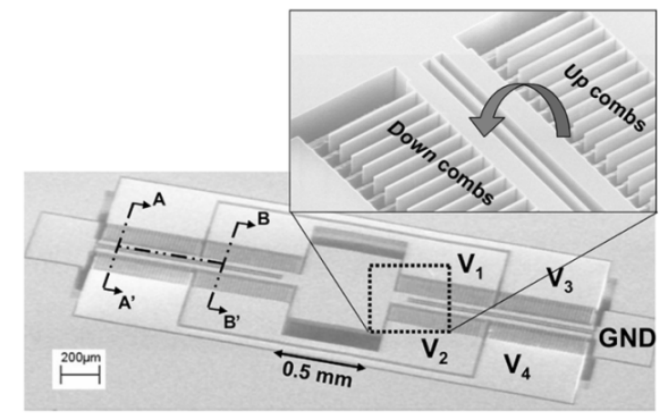

(a)

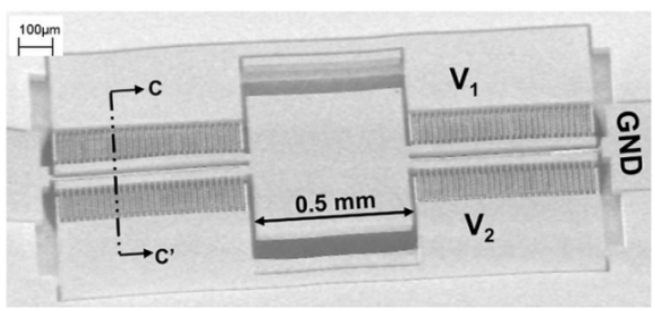

(b)

Fig. 1. Scanning electron micrographs of fabricated micromirrors: (a) Device 1 with four isolated vertical comb drive sets for up and down piston motion as well as independent bidirectional rotation. (b) Device 2 with two isolated comb drive sets for bidirectional rotation.

connect to the common shuttle, and are, therefore, also electrically and mechanically coupled. As a result, only downward motion is possible, and rotation is always coupled with vertical and lateral motion of the shuttle. Also, the upper and lower comb-finger sets are separated by the thickness of insulating oxide $(\sim 1 \mu \mathrm{m})$, requiring large biasing (pretilting) of devices before the comb-fingers are adequately engaged. Preengagement of vertical comb-fingers is highly desirable for well-behaved performance at lower actuation voltages [13]. This was previously demonstrated in a silicon optical scanner fabricated by eutectic bonding assembly [14], as well as in the electrostatic vertical comb actuator fabricated in the BELST II process [15].

Our devices are fabricated in a four-mask SOI-MEMS process that alleviates the above limitations. Namely, 1) all comb-fingers are fabricated in the device layer allowing isolated independently powered vertical comb drive sets. This enables independent up- or down-pistoning and bidirectional rotation as demonstrated by Device 1 in this work.2)Comb-fingersaretimedetched such that there is several microns of preengagement (overlap) allowing constant force operation from 0 V. 3) Support beams can be of any desired thickness for lower-voltage operation, $30-\mu \mathrm{m}$ upper beams (of a $50-\mu \mathrm{m}$-thick device layer) in Device 1 , or the more compliant $10-\mu$ $\mathrm{m}$-thick middle beams in Device 2.4) Masks for etching of combfingers are self-aligned by a single mask. 5) Devices are made in monolithic single-crystal silicon for repeatable and reliable operation. 


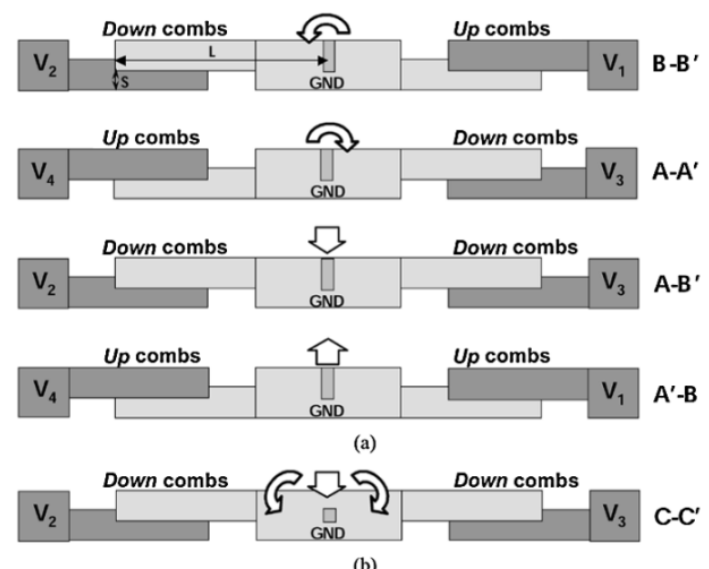

(b)

Fig. 2. Schematics of various device cross sections and their resulting actuation. (a) Four possible comb drive set arrangements in Device 1. In the center, the structure is suspended by the upper beam. (b) Comb drive arrangement for Device 2 which demonstrates bidirectional rotation and/or downward pistoning.

\section{DESIGN CONSIDERATIONS}

The multilevel beam SOI-MEMS methodology allows the designer for the first time to utilize any number of fully isolated sets of large static stroke vertical comb drives, where some can be up actuating and some down actuating. The variety of possible beams and actuators gives the designer a great deal of freedom to meet device specifications. In our designs, both the up-actuating and down-actuating comb drives are utilized (Figs. 1 and 2) as follows. For best rotation performance, or pure rotation, we utilize a down comb drive set and an up comb drive set, each on opposing sides of the rotation axis, shown in Fig. 2(a) cross section B-B' [also Fig. 1(a)]. Specifically, that is achieved by applying equivalent dc actuation voltage to electrodes $V_{1}$ and $V_{2}$, and grounding electrodes (GND), $V_{3}$ and $V_{4}$. Opposing comb-fingers are, thus, vertically pulled in by electrostatic force which aims to increase capacitance between the comb-fingers by increasing overlap-area. Increase in voltage, thereby, increases angle of rotation until the overlap area is maximized, which occurs when the moving comb-finger edge reaches the edge of the static comb-finger. Maximum angle of rotation can, therefore, be approximated by simply knowing the distance of the moving comb-finger edge from rotation axis $L$ and the maximum stroke $S$ [Fig. 1(a)] as $\theta \approx \operatorname{atan}(S / L)$, which is approximately $S / L$ for small angles.

For rotation in the opposite direction, and thus doubling the overall range of static rotation, another such pair of comb drive sets is utilized, but on reverse sides as in cross section A-A' of Fig. 2(a). In this case, $V_{3}$ and $V_{4}$ are enabled, $V_{1}$ and $V_{2}$ grounded. Thus, with four comb drive sets as shown in Device 1 [Fig. 1(a)], two on each side of the axis and two on the opposite side, bidirectional rotation is achieved. This capability trades off with higher actuation voltage, however. Namely, for performance where one-sided rotation angle (e.g., $0^{\circ}$ to $10^{\circ} \mathrm{me}-$ chanical rotation) is adequate, only two sets of comb drives are needed, and thus, given the same device area, twice the number of comb-fingers would be utilized in each set.

Since all four comb drive sets shown in Fig. 1(a) have independent voltage control, labeled as electrodes $V_{1}, V_{2}, V_{3}$, and $V_{4}$, it should be noted that two more cross sections are possible by activating diagonally opposing sets. Activating $V_{2}$ and $V_{3}$ and

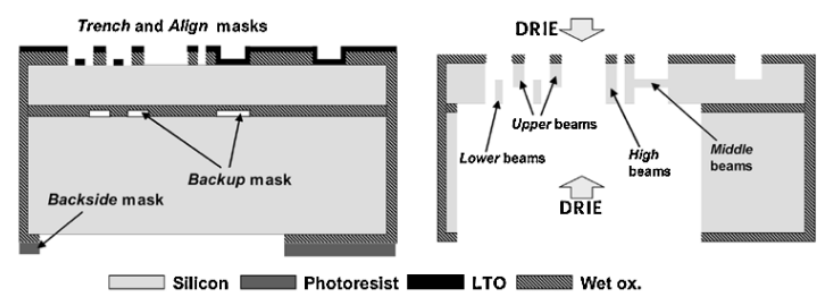

(a)
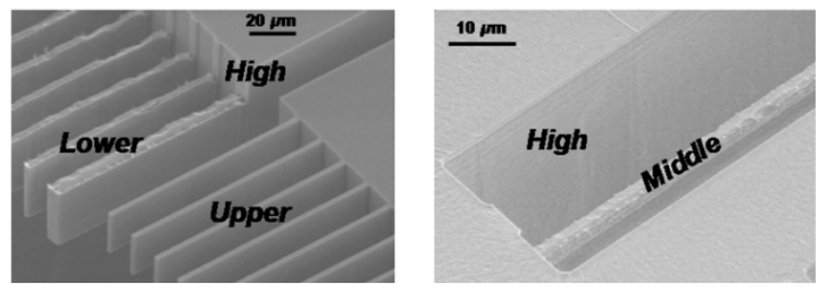

(b)

Fig. 3. Fabrication process schematic and results, (a) on the left is the SOI wafer after all four masks have been applied to it, and on the right is a schematic of the same wafer cross section after both the back side and front side DRIE steps are completed. (b) Resulting multilevel beam structures that form suspensions, vertical comb drives, etc. In the left image, lower beams show roughness due to the timed-etch fabrication.

grounding $V_{1}$ and $V_{4}$, as shown in cross section A-B' (Figs. 1 and 2), gives downward pistoning, and finally, activating $V_{1}$ and $V_{4}$ and grounding $V_{2}$ and $V_{3}$ (cross section A'-B Figs. 1 and 2), gives upward pistoning.

\section{MultileVEL BEAm SOI-MEMS FABRICATION}

The four design layers explained in Section II are depicted in a wafer cross section in Fig. 3(a) and shown fabricated in Fig. 3(b). In addition to these four layers, the back side of the device should be opened to allow large rotation or piston motion and dry releasing of the device. The multilevel beam SOIMEMS process utilizes multilevel etching of the SOI device layer from front and back side by deep reactive ion etch (DRIE) [16]. It has been demonstrated for laterally driven torsional micromirror application [5].

The advanced version of that process, utilized in this work, is described in more detail elsewhere [16], and schematically depicted in Fig. 3. The critical improvement from [5] is the preetching of the Backup mask in buried isolation oxide layer during SOI wafer bonding which enables more accurate definition of Upper features as well as minimum feature distance between various beam types. Namely, in the advanced process, the two critical, beam-defining masks (effectively one for Upper fingers, and one for Lower fingers) are prepared from the top side using the wafer-stepper for accurate alignment, and are both aligned to the buried Backup mask (the buried mask is exposed by top-side etching in two places on the wafer to allow alignment to it). Another feature of the process is the mask self-alignment, which is critical capability for useful vertical comb drive based devices. Namely, as detailed in [16], the first top-side mask Trench is intentionally overgrown by a small margin such that the second mask, Align used to outline Lower beams, corrects the features of the previous mask to designed dimensions which effectively aligns all of the top-side features to that single Align mask. All the masks are, therefore, in place before DRIE steps are started.

The DRIE steps are timed such that back side DRIE into the Backup mask creates Upper beams, while front side timed DRIE 

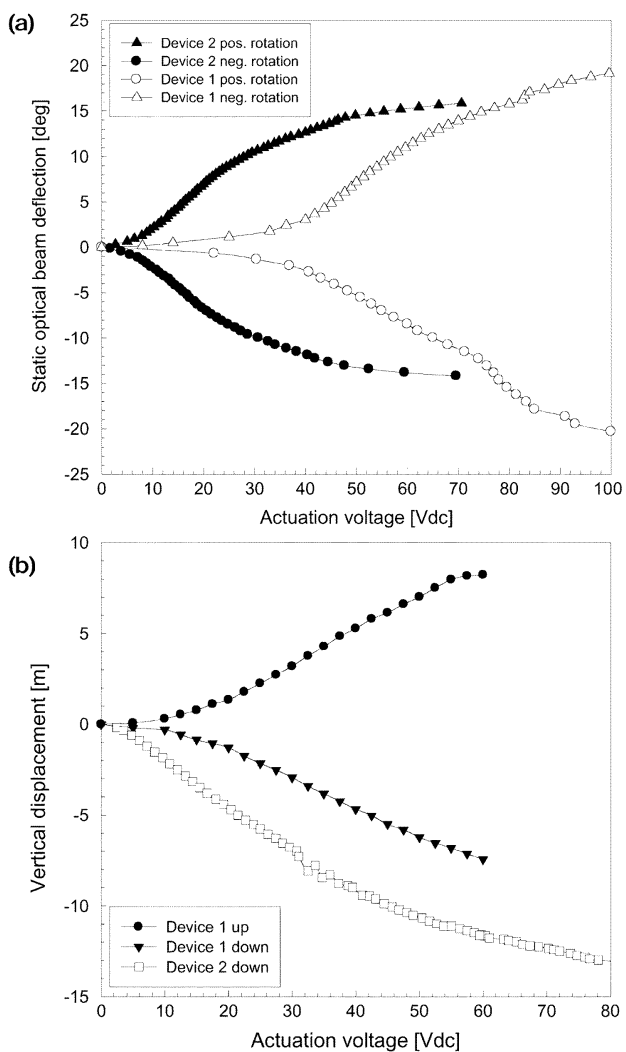

Fig. 4. Characterization of the two devices: (a) dc actuation voltage versus static optical deflection of a laser beam-Device 2 operates at lower voltages due to the more compliant middle support beam, and (b) dc actuation voltage versus static pistoning actuation (out of wafer plane). Device 2 has downward pistoning only, as shown in Fig. 2(b).

defines the Lower beams. Where both etches are applied, Middle beams result. Full thickness of the SOI device layer is $50 \mu \mathrm{m}$, Lower beams were fabricated to reach from 0 to $30 \mu \mathrm{m}$, while Upper beams from 20 to $50 \mu \mathrm{m}$, thus leaving $10 \mu \mathrm{m}$ of overlap which is also, therefore, the thickness of Middle beam utilized in Device 2.

It should be noted that actual fabricated beam thicknesses vary across the wafer due to the timed etches that define thicknesses of Upper and Lower beams have some nonuniformity across the wafer, usually up to $3 \%-5 \%$ of the $20-\mu \mathrm{m}$ etch, resulting in beam thicknesses of $30 \pm 1 \mu \mathrm{m}$.

\section{Characterization AND Discussion}

The two types of devices were tested for static optical deflection as a function of a voltage on a simple optical test setup by measuring optical deflection of a laser beam under a varying $\mathrm{dc}$ bias from 0 to over $100 \mathrm{~V}$. The results are recorded in Fig. 4(a). The device exhibits large static deflections as expected from the design, given parameters $L=110 \mu \mathrm{m}$ and $S=20 \mu \mathrm{m}$, as discussed in Section II. Device 1 operates at lower voltages as expected due to the more compliant support beams. It can also be noted in Fig. 4(a) that Device 1 has a very small deflection until $\sim 30 \mathrm{~V}$. It was later determined that that particular device did not have the full $10-\mu \mathrm{m}$ comb-finger preengagement due to some overetching in that part of the wafer. The comparison of the two graphs, therefore, demonstrates the advantage of preengagement and also shows the need for improved process control.
Bode plots were obtained using a laser doppler velocimeter, at $10-\mathrm{V}$ bias, and small-signal ac voltage. In pistoning mode, Device 1 exhibits resonance at $2619 \mathrm{~Hz}$ while in rotation mode at $1491 \mathrm{~Hz}$. The significantly higher pistoning mode resonance was expected since the support beams have a large thickness giving cubic relation to vertical deflection stiffness. Measurements of the radii of curvature (RoC) of individual mirrors (no metal reflection coating) were obtained using a Veeco white light interferometer. The $\mathrm{RoC}$ in all cases were greater than the measurement capability of the interferometer $(>5 \mathrm{~m})$.

\section{CONCLUSION}

The combination of high-performance vertical comb drives with up-actuation and down-actuation has been demonstrated to add new DoF to SOI-MEMS, greatly increasing its range of applications.

\section{REFERENCES}

[1] S. Blackstone and T. Brosnihan, "SOI MEMS technologies for optical switching," in Optical MEMS 2001, Okinawa, Japan, Sept. 2001.

[2] R. Conant, J. Nee, K. Y. Lau, and R. S. Muller, "A flat high-frequency scanning micromirror," in Proc. Solid-State Sensor Actuator Workshop, Hilton Head, SC, June 4-8, 2000, pp. 6-9.

[3] J. T. Nee, R. A. Conant, R. S. Muller, and K. Y. Lau, "Lightweight, optically flat micromirrors for fast beam steering," in 2000 IEEE/LEOS Int. Conf. Optical MEMS, Kauai, HI, Aug. 21-24, 2000, pp. 9-10.

[4] W. Noell, P.-A. Clerc, L. Dellmann, B. Guldimann, H.-P. Herzig, O. Manzardo, C. R. Marxer, K. J. Weible, R. Dandliker, and N. de Rooij, "Applications of SOI-based optical MEMS," IEEE J. Select. Topics Quantum Electron., vol. 8, pp. 148-154, Jan./Feb. 2002.

[5] V. Milanović, M. Last, and K. S. J. Pister, "Torsional micromirrors with lateral actuators," in Transducers 2001, Muenchen, Germany, June 2001.

[6] V. Milanović, M. Last, and K. S. J. Pister, "Monolithic silicon micromirrors with large scanning angle," in 2001 IEEE/LEOS Int. Conf. Optical MEMS, Okinawa, Japan, Sept. 2001.

[7] G.-D. J. Su, H. Toshiyoshi, and M. C. Wu, "Surface-micromachined 2-D optical scanners with high-performance single-crystalline silicon micromirrors," IEEE Photon. Technol. Lett., vol. 13, pp. 606-608, June 2001.

[8] U. Krishnamoorthy and O. Solgaard, "Self-aligned vertical comb-drive actuators for optical scanning micromirrors," in 2001 IEEE/LEOS Int. Conf. Optical MEMS, Okinawa, Japan, Sept. 2001.

[9] T. Overstolz, P.-A. Clerc, M. T. Gale, H. P. Herzig, G. Niederer, W. Noell, J. Soechtig, H. Thiele, and N. F. de Rooij, "Tilting out-of-plane platform for optical applications," in 2002 IEEE/LEOS Int. Conf. Optical MEMS, Lugano, Switzerland, Aug. 2002, pp. 81-82.

[10] Y. Mizuno, O. Tsuboi, N. Kouma, H. Soneda, H. Okuda, Y. Nakamura, S. Ueda, I. Sawaki, and F. Yamagishi, "A 2-axis comb-driven micromirror array for 3D MEMS switches," in 2002 IEEE/LEOS Int. Conf. Optical MEMS, Lugano, Switzerland, Aug. 2002, pp. 17-18.

[11] U. Krishnamoorthy, K. Li, K. Yu, D. Lee, J. P. Heritage, and O. Solgaard, "Dual-mode micromirrors for optical phased array applications," in Transducers 2001, Munich, Germany, June 2001.

[12] A. P. Lee, C. F. McConaghy, P. A. Krulevitch, E. W. Campbell, G. E. Sommargren, and J. C. Trevino, "Electrostatic comb drive for vertical actuation," in Proc. SPIE-Int. Society Optical Engineering, vol. 3224, 1997, pp. 109-119.

[13] J.-L. A. Yeh, C.-Y. Hui, and N. C. Tien, "Electrostatic model for an asymmetric vertical comb-drive," J. Microelectromechanical Systems, vol. 9, no. 1, Mar. 2000.

[14] J.-M. Kim, Y.-C. Ko, D.-H. Kong, J.-M. Kim, K. B. Lee, and D.-Y. Jeon, "Fabrication of silicon optical scanner for laser display," in 2000 IEEE/LEOS Int. Conf. Optical MEMS, Kauai, HI, Aug. 21-24, 2000, pp. $13-14$.

[15] J. M.-L. Tsai, H.-Y. Chu, J. Hsieh, and W. Fang, "The BELST II process for a silicon high-aspect-ratio micromaching vertical comb actuator and its applications," J. Micromech. Microeng., vol. 14, pp. 235-241, Nov. 2003.

[16] V. Milanović, "Multilevel-beam SOI-MEMS fabrication and applications," J. Microelectromechanical Systems, vol. 13, no. 1, pp. 19-30, Feb. 2004. 